\title{
BMJ Open Prevalence and patterns of congenital heart diseases in Africa: a systematic review and meta-analysis protocol
}

\author{
Aurel T Tankeu, ${ }^{1}$ Jean Joel R Bigna, ${ }^{2,3}$ Jobert Richie N Nansseu, ${ }^{4,5}$ \\ Leopold Ndemnge Aminde, ${ }^{6}$ Celestin Danwang, ${ }^{7}$ Mazou N Temgoua, ${ }^{1}$ \\ Jean Jacques N Noubiap ${ }^{8}$
}

To cite: Tankeu AT, Bigna JJR, Nansseu JRN, et al. Prevalence and patterns of congenital heart diseases in Africa: a systematic review and meta-analysis protocol. BMJ Open 2017;7:e015633. doi:10.1136/bmjopen-2016015633

- Prepublication history and additional material is available. To view please visit the journal (http://dx.doi.org/ 10.1136/bmjopen-2016015633).

Received 19 December 2016 Revised 18 January 2017 Accepted 19 January 2017

CrossMark

For numbered affiliations see end of article.

Correspondence to Dr Jean Jacques N Noubiap; noubiapjj@yahoo.fr

\section{ABSTRACT}

Introduction: Congenital heart diseases (CHD) are common causes of cardiovascular morbidity and mortality among young children and adolescents living in Africa. Accurate epidemiological data are needed in order to evaluate and improve preventive strategies. This review aims to determine the prevalence of $\mathrm{CHD}$ and their main patterns in Africa.

Methods and analysis: This systematic review and meta-analysis will include cross-sectional, case-control and cohort studies of populations residing inside African countries, which have reported the prevalence of $\mathrm{CHD}$, confirmed by an echocardiographic examination and/or describing different patterns of these abnormalities in Africa. Relevant abstracts published without language restriction from 1 January 1986 to 31 December 2016 will be searched in PubMed, Exerpta Medica Database and online African journals as well as references of included articles and relevant reviews. Two review authors will independently screen, select studies, extract data and assess the risk of bias in each study. The study-specific estimates will be pooled through a random-effects meta-analysis model to obtain an overall summary estimate of the prevalence of CHD across studies. Clinical and statistical heterogeneity will be assessed, and we will pool studies judged to be clinically homogeneous. On the other hand, statistical heterogeneity will be evaluated by the $\chi 2$ test on Cochrane's $Q$ statistic. Funnel-plots analysis and Egger's test will be used to detect publication bias. Results will be presented by geographic region (central, eastern, northern, southern and western Africa).

Ethics and dissemination: The current study will be based on published data, and thus ethical approval is not required. This systematic review and meta-analysis is expected to serve as a base which could help in estimating and evaluating the burden of these abnormalities on the African continent. The final report of this study will be published in a peer-reviewed journal.

Trial registration number: PROSPERO CRD42016052880.

\section{Strengths and limitations of this study}

- To the best of our knowledge, this will be the first systematic review on the topic of congenital heart diseases (CHD) to summarise available data on the African continent.

- We will use powerful meta-analysis techniques to derive accurate estimates.

- A major possible limitation of this study could be the limited data with predominance of hospital-based studies. Indeed, these studies may not reflect the true prevalence of CHD in the general population, therefore overestimating these estimates.

- Another possible limitation may be heterogeneity of studies carried out on the topic in Africa.

\section{INTRODUCTION}

Congenital heart disease or congenital heart defect (CHD) is a problem of the heart's structure and function present at birth, affecting the heart or adjacent great blood vessels, detected either at the time of birth or detected later in life. ${ }^{1}{ }^{2}$ Worldwide, CHD are the main heart diseases found in children and constitute one of the major causes of infant mortality, particularly in developing countries. $^{2}{ }^{3}$ They also represent the most common of all congenital malformations accounting for more than $20 \%$ of perinatal deaths. ${ }^{45}$ Their estimated prevalence is eight cases per 1000 live births across the globe, representing $\sim 1.35$ million newborns each year with CHD, but these figures vary worldwide. ${ }^{2}$ For instance, the incidence of CHD in different studies varies from about 4/1000 to $50 / 1000$ live births and, despite advances in detection and treatment, congenital heart diseases account for $3 \%$ of all infant deaths and $46 \%$ of deaths from congenital malformations in developed countries such as the 
USA. $^{4-6}$ In addition, these abnormalities can be lifethreatening in early childhood, and children born with severe forms are at $\sim 12$ times higher risk of mortality in the first year of life. ${ }^{7}$ Thus, hundreds of thousands of children die each year from CHD, while millions more remain in desperate need of treatment in the developing world. ${ }^{8}$ Since mortality and morbidity from cardiac disease among children in developing countries are gaining recognition, there is a need of summarised data on CHD in the African continent. Africa is thought to have one of the highest prevalences of heart diseases in children and young adults, including CHD, but the main findings include evidence that the CHD burden is underestimated mainly due to the poor outcome of African children with $\mathrm{CHD}^{7}$ From a global point of view, the epidemiology of these abnormalities is still unknown in Africa with few data on the topic. Reducing the prevalence of these diseases is urgent and requires a real inventory of the premises of the problem that would clarify the issue for more effective prevention strategies and improved management. In this context, we present the protocol for a systematic review and meta-analysis to assess the prevalence and patterns of CHD in Africa.

\section{OBJECTIVE}

This systematic review and meta-analysis aims to determine the prevalence of CHD and their different patterns in Africa.

\section{Review question}

This review of studies published in the past 30 years, from 1 January 1986 to 31 December 2016, should answer the following questions:

1. What is the prevalence of CHD among African populations?

2. What are the different patterns of CHD in Africa?

\section{METHODS AND ANALYSIS}

Criteria for considering studies for the review

Inclusion criteria

We will include:

1. Cross-sectional, case-control or cohort studies of populations residing in African countries reporting the prevalence of CHD in African countries or enough data to compute these estimates, regardless of stillbirth.

2. Studies describing the different patterns of CHD on the African continent.

\section{Exclusion criteria}

We will not consider:

1. Studies on congenital abnormalities involving the heart but not considered as CHD (Long QT syndrome, congenital but functionless abnormalities of the heart, cardiomyopathies).

2. Studies conducted among populations of African origin residing outside Africa.
3. Studies in subgroups of participants selected on the basis of cardiac murmurs without echocardiographic confirmation of CHD (eg, suspected but nonconfirmed CHD).

4. Studies including many pathologies in which it will not be possible to extract data regarding CHD or studies reporting congenital abnormalities affecting many systems at a time.

5. Letters, reviews, commentaries and editorials.

6. Studies lacking key data and/or explicit method description.

7. Duplicates: for studies published in more than one paper, the most comprehensive one reporting the largest sample size will be considered.

8. Studies whose full data will not be accessible even after request from the authors.

\section{Search strategy for identifying relevant studies}

The search strategy will be implemented in two stages:

\section{Bibliographic database searches}

(A) Relevant abstracts published without language restriction on the prevalence, incidence, main aetiologies and clinical features of CHD in Africa will be identified via a search of PubMed, Exerpta Medica Database and online African journals. The search will be limited to studies published between 1 January 1986 and 31 December 2016. Both text words and medical subject heading terms will be used. Key search terms include: 'congenital heart defects' or 'congenital heart disease' as well as the name of every known pattern of these abnormalities. We will also use individual country names for the 54 African countries as additional key search terms for more abstracts on the subject. Conference proceedings and studies from grey literature of the study period will also be identified through databases and checked. The main search strategy is shown in table 1.

(B) The abstracts of all eligible papers will be reviewed and full articles will be accessed through PubMed, Exerpta Medica Database, Google Scholar, HINARI or journals' websites. Additionally, references of all relevant articles and reviews will be scrutinised for other potential data sources and their full texts will be accessed in a similar way. The authors whose full text papers will not be accessible by the numerous internet-based sources will be directly contacted to provide them. In case of no feedback from these authors, the corresponding studies will be excluded.

\section{Searching for other sources}

References of all relevant researches and review articles will be scrutinised for additional potential data sources, and their full texts will be accessed in a similar way. Those authors whose full text papers will not be accessible by the numerous internet-based sources will be directly contacted via email to provide them. In case of no 
Table 1 Search strategy for PubMed

\begin{tabular}{|c|c|}
\hline $\begin{array}{l}\text { Search } \\
\text { (numbers) }\end{array}$ & Search terms \\
\hline 1 & $\begin{array}{l}\text { congenital heart OR heart defects OR heart murmur OR fallot OR ventricular defect OR atrial defect OR septal } \\
\text { defect OR great vessels transposition OR foramen ovale OR single ventricle OR persistent ductus arteriosus OR } \\
\text { dorv OR avsd OR ASD OR VSD OR BAV OR d-tga OR aorta coarctation OR hypoplastic heart OR total } \\
\text { anomalous pulmonary venous connection OR truncus arteriosus OR Ebstein's abnormality OR tricuspid atresia } \\
\text { OR cyanotic heart OR non cyanotic heart OR mitral stenosis[tw] OR mitral incompetence[tw] OR tricuspid } \\
\text { incompetence[MeSH terms] OR tricuspid stenosis[tw] OR pulmonary stenosis[tw] OR pulmonary incompetence } \\
\text { [tw] OR aortic incompetence[tw] OR aortic stenosis[MeSH terms] OR heart murmur[tw] OR Atrial septal defect } \\
\text { [tw] OR Ventricular septal defect[tw] OR Pulmonary atresia[tw] OR Aortic atresia[tw] OR echocardiography } \\
\text { abnormalities[tw] OR Fallot tetralogy[tw] OR aorta coarctation[tw] OR cyanotic cardiac abnormalities[tw] OR } \\
\text { atrioventricular septal defects[tw] OR biscupid aortic valve[tw] OR double outlet right ventricle[tw] OR patent } \\
\text { ductus arteriosus[tw] OR single ventricle[tw] OR great arteries transposition[tw] OR hypoplastic heart[tw] OR } \\
\text { tricuspid atresia[tw] OR Ebstein abnormality[tw] OR truncus arteriosus[tw] OR total anomalous pulmonary } \\
\text { venous connection[tw] }\end{array}$ \\
\hline 2 & $\begin{array}{l}\text { (Africa* OR Algeria OR Angola OR Benin OR Botswana OR "Burkina Faso" OR Burundi OR Cameroon OR } \\
\text { "Canary Islands" OR "Cape Verde" OR "Central African Republic" OR Chad OR Comoros OR Congo OR } \\
\text { "Democratic Republic of Congo" OR Djibouti OR Egypt OR "Equatorial Guinea" OR Eritrea OR Ethiopia OR } \\
\text { Gabon OR Gambia OR Ghana OR Guinea OR "Guinea Bissau" OR "Ivory Coast" OR "Cote d'Ivoire" OR } \\
\text { Jamahiriya OR Kenya OR Lesotho OR Liberia OR Libya OR Madagascar OR Malawi OR Mali OR Mauritania } \\
\text { OR Mauritius OR Mayotte OR Morocco OR Mozambique OR Namibia OR Niger OR Nigeria OR Principe OR } \\
\text { Reunion OR Rwanda OR "Sao Tome" OR Senegal OR Seychelles OR "Sierra Leone" OR Somalia OR "South } \\
\text { Africa" OR "South Sudan" OR "St Helena" OR Sudan OR Swaziland OR Tanzania OR Togo OR Tunisia OR } \\
\text { Uganda OR "Western Sahara" OR Zaire OR Zambia OR Zimbabwe OR "Central Africa" OR "Central African" OR } \\
\text { "West Africa" OR "West African" OR "Western Africa" OR "Western African" OR "East Africa" OR "East African" } \\
\text { OR "Eastern Africa" OR "Eastern African" OR "North Africa" OR "North African" OR "Northern Africa" OR } \\
\text { "Northern African" OR "South African" OR "Southern Africa" OR "Southern African" OR "sub Saharan Africa" OR } \\
\text { "sub Saharan African" OR "subSaharan Africa" OR "subSaharan African") NOT ("guinea pig" OR "guinea pigs" } \\
\text { OR "aspergillus niger") }\end{array}$ \\
\hline 3 & $\# 1$ AND \#2 \\
\hline 4 & \# 3 Limits: : $1986 / 01 / 01$ to $2016 / 12 / 31$ \\
\hline
\end{tabular}

feedback from these authors, the corresponding studies will be excluded.

\section{Selection of studies for inclusion in the review}

Assessment of eligible papers will be independently carried out by two authors using an assessment guide to ensure that the selection criteria are reliably applied by all the team members. They will screen the titles and abstracts obtained from the searches and retrieve the full texts of potentially eligible papers by at least one author. Thereafter, they will independently review the full text of each potentially eligible study, compare their results and resolve any discrepancy by discussion and consensus. If a decision is not reached, a third review author will be consulted for arbitration.

\section{Assessment of methodological quality and reporting of data}

The Newcastle-Ottawa Scale for assessing the quality of non-randomised studies in meta-analyses will be used to assess the methodological quality and risk of bias for each study (see online supplementary appendix S1). ${ }^{9}$ Risk of bias and quality scores will be presented in a table.

\section{Data extraction and management}

A data extraction sheet will be used to collect information about the author, the country, the year of publication, the study design, the sample size population, the mean/median age of the population, the age range, the sex ratio, the prevalence of CHD as well as main patterns when available. Where prevalence or information for calculating them (eg, sample size, number of outcomes) is lacking, we will directly contact the corresponding author and request the information. In case of multinational studies, we will separate the results to show the prevalence and aetiologies within individual countries. Where it will not be possible to disaggregate the data by country, the study will be presented as one and the countries in which the study was carried out will be shown.

\section{Data synthesis and analysis}

Data will be analysed using Stata software V.13 (Stata Corp V.13, Texas, USA). A meta-analysis will be conducted for data obtained from studies in which CHD has the same aetiologies, and SEs for the study-specific estimates will first be determined from the point estimate and the appropriate denominators, assuming a binominal distribution. Then the study-specific estimates will be 
pooled through a random-effects meta-analysis model to obtain an overall summary estimate of the prevalence across studies, after stabilising the variance of individual studies using the Freeman-Tukey double arc-sine transformation (to keep the effect of studies with extremely small or large estimates). ${ }^{10}$ Heterogeneity will be evaluated by the $\chi^{2}$ test on Cochrane's $Q$ statistic ${ }^{11}$ which is quantified by $\mathrm{I}^{2}$ values, assuming that $\mathrm{I}^{2}$ values of $25 \%$, $50 \%$ and $75 \%$ represent low, medium and high heterogeneity, respectively. ${ }^{12}$ Where substantial heterogeneity will be detected, a subgroup analysis will be performed to detect its possible sources using the following grouping variables: type of $\mathrm{CHD}$, consequences on cardiac haemodynamic, study setting (hospital vs communitybased), age of diagnosis, geographical area (central, eastern, northern, southern and western Africa) and study quality. Difference between groups will be detected if $p$ value $<0.05$. Inter-rater agreement for study inclusion will be assessed using Cohen's $\kappa$ coefficient. ${ }^{13}$ Funnel plots analysis and Egger's test ${ }^{14}$ will be performed to detect publication bias. Publication bias will be confirmed if $p$ value on Egger's test $<0.10$. Results will be presented by geographic region (central, eastern, northern, southern and western Africa).

\section{PRESENTATION AND REPORTING OF RESULTS}

The study selection process will be summarised using a flow diagram. Reasons for studies' exclusion will be described. The report will follow the Preferred Reporting Items for systematic review and Meta-analysis (PRISMA) Guidelines. ${ }^{15}$ Tables and forest plots will serve to summarise quantitative data where appropriate. We will examine prevalence and aetiologies by region, setting (hospital or community) and time period depending on the data available. We plan to report on quality scores and risk of bias for each eligible study. This may be tabulated and accompanied by narrative summaries.

\section{CONCLUSION}

CHD represent the second major cause of cardiovascular disease morbidity and mortality among young Africans. Their management is limited in Africa in an inadequate socioeconomic environment with an insufficient technical platform and human resources. Moreover, the global burden of these conditions is unknown on the continent. Therefore, it becomes urgent to provide summarised recent and reliable data in order to help estimate the prevalence as well as aetiologies in Africa in order to highlight the importance of these abnormalities and the need for reinforcement of effective preventive strategies based on systematic screening of cardiac abnormalities in the perinatal period. We hope that this review will help sensitise healthcare providers on the problem represented by CHD in Africa, assist and support the implementation of new policies, practices and researches by providing insights into the current situation and the true impact of CHD on the African continent as well as shortcomings that can guide future research around this topic.

\section{Protocol and registration}

The protocol for this review has been published in the PROSPERO International Prospective Register of systematic reviews (http://www.crd.york.ac.uk/PROSPERO), registration number: PROSPERO CRD42016052880.

\section{Author affiliations}

${ }^{1}$ Department of Internal Medicine and Specialties, Faculty of Medicine and Biomedical Sciences, Yaoundé, Cameroon

${ }^{2}$ Department of Epidemiology and Public Health, Centre Pasteur of Cameroon, Yaoundé, Cameroon

${ }^{3}$ Faculty of Medicine, University of Paris Sud XI, Le Kremlin Bicêtre, France ${ }^{4}$ Department of Public Health, Faculty of Medicine and Biomedical Sciences, University of Yaoundé 1, Yaoundé, Cameroon

${ }^{5}$ Sickle Cell Disease Unit, Mother and Child Centre of the Chantal Biya Foundation, Yaoundé, Cameroon

${ }^{6}$ Faculty of Medicine \& Biomedical Sciences, School of Public Health, The University of Queensland, Brisbane, Queensland, Australia

${ }^{7}$ Department of Surgery and Specialties, Faculty of Medicine and Biomedical Sciences, University of Yaoundé 1, Yaoundé, Cameroon

${ }^{8}$ Department of Medicine, Groote Schuur Hospital and University of Cape Town, Cape Town, South Africa

Contributors ATT and JJNN conceived and designed the protocol. ATT drafted the manuscript. JJRB, JRNN, LNA, CD, TNM and JJNN critically revised the manuscript for methodological and intellectual content. JJNN is the guarantor of the review. All authors approved the final version of this manuscript.

Funding This research received no specific grant from any funding agency in the public, commercial or not-for-profit sectors.

Competing interests None declared

Provenance and peer review Not commissioned; externally peer reviewed.

Open Access This is an Open Access article distributed in accordance with the Creative Commons Attribution Non Commercial (CC BY-NC 4.0) license, which permits others to distribute, remix, adapt, build upon this work noncommercially, and license their derivative works on different terms, provided the original work is properly cited and the use is non-commercial. See: http:// creativecommons.org/licenses/by-nc/4.0/

\section{REFERENCES}

1. Tantchou Tchoumi JC, Butera G, Giamberti A, et al. Occurrence and pattern of congenital heart diseases in a rural area of sub-Saharan Africa. Cardiovasc J Afr 2011;22:63-6.

2. Chelo D, Nguefack F, Menanga AP, et al. Spectrum of heart diseases in children: an echocardiographic study of 1,666 subjects in a pediatric hospital, Yaounde, Cameroon. Cardiovasc Diagn Ther 2016;6:10-19.

3. Kouame BD, N'guetta-Brou IA, Kouame GSY, et al. Epidemiology of congenital abnormalities in West Africa: results of a descriptive study in teaching hospitals in Abidjan: Cote d'Ivoire. Afr J Paediatr Surg 2015;12:51-5.

4. Sadowski SL. Congenital cardiac disease in the newborn infant: past, present, and future. Crit Care Nurs Clin North Am 2009;21:37-48.

5. Hoffman JIE, Kaplan S. The incidence of congenital heart disease. J Am Coll Cardiol 2002;39:1890-900.

6. Chinawa JM, Eze JC, Obi I, et al. Synopsis of congenital cardiac disease among children attending University of Nigeria Teaching Hospital Ituku Ozalla, Enugu. BMC Res Notes 2013;6:475.

7. Otaigbe BE, Tabansi PN. Congenital heart disease in the Niger Delta region of Nigeria: a four-year prospective echocardiographic analysis. Cardiovasc J Afr 2014;25:265-8. 
8. Massoure PL, Roche NC, Lamblin G, et al. Cardiovascular disease in children in Djibouti: a single-centre study. Pan Afr Med J 2013; $14: 141$.

9. Turner L, Boutron I, Hróbjartsson A, et al. The evolution of assessing bias in Cochrane systematic reviews of interventions: celebrating methodological contributions of the Cochrane Collaboration. Syst Rev 2013;2:79.

10. Miller JJ. The inverse of the Freeman-Tukey double Arcsine transformation. Am Stat 1978;32:138.

11. Cochran GW. The combination of estimates from different experiments. Biometrics 1954;10:101-29.
12. Huedo-Medina TB, Sánchez-Meca J, Marín-Martínez F, et al. Assessing heterogeneity in meta-analysis: q statistic or 12 index? Psychol Methods 2006;11:193-206.

13. McHugh ML. Interrater reliability: the kappa statistic. Biochem Medica 2012;22:276-82.

14. Egger M, Davey Smith G, Schneider M, et al. Bias in metaanalysis detected by a simple, graphical test. BMJ 1997;315: 629-34.

15. Moher D, Liberati A, Tetzlaff J, et al. Preferred reporting items for systematic reviews and meta-analyses: the PRISMA statement. J Clin Epidemiol 2009;62:1006-12. 